\title{
Hydrodynamics, probability and the geometry of the diffeomorphisms group
}

\author{
Ana Bela Cruzeiro
}

\begin{abstract}
We characterize the solution of Navier-Stokes equation as a stochastic geodesic on the diffeomorphisms group, thus generalizing Arnold's description of the Euler flow.

Mathematics Subject Classification (2000). Primary 37L55; Secondary 35Q30, 58E30, 58J65, 60J60, 76D05.

Keywords. Navier-Stokes equation; diffeomorphisms group; stochastic geodesic equation.
\end{abstract}

\section{Introduction}

Laplacian canonical determinism (quoting Laplace himself "We may regard the present state of the universe as the effect of its past and the cause of its future...") has been called into question by the studies of H. Poincaré and J. Hadamard of complex highly unstable trajectories of a system of three bodies moving under the effect of gravitation forces. Much later E. Lorenz considered some simplified model for atmospheric convection with a small number of degrees of freedom and also found chaotic motions, the term chaotic meaning that small changes in the initial conditions produce big perturbations in the behaviour of the trajectories of motion (sometimes popularly referred to as the "butterfly effect").

Dynamics may therefore exibit a chaotic (random?) behaviour even when the governing equations are deterministic. Beyond classical dynamics, the most convincing illustration of this is, of course, quantum mechanics.

In Turbulence, modelized by the (deterministic) incompressible Navier-Stokes equation

$$
\frac{\partial u}{\partial t}+u . \nabla u=\nu \Delta u-\nabla p, \operatorname{div} u=0, u(0, x)=u_{0}(x)
$$

This work was completed with the support of the Fundação para a Ciência e a Tecnologia through the project PTDC/MAT/69635/2006. 
with small viscosity coefficient $\nu>0$, such classical chaotic phenomena really occur. On the other hand statistical effects also manifest themselves: they are called coherent structures (for example the Jupiter's red spot or Açores anticyclone). We refer to [15] for an interesting discussion of these problems.

There are various possible reasons to look for probabilistic content in Fluid Dynamics. The obvious observations that initial conditions are difficult to know precisely and that the observable computed in practice have to be statistical quantities points out to some statistical approach, found, for example, in works of Vishik, Fursikov, Foias, etc. ([10]).

One can of course add some noise to given deterministic systems and study the randomly perturbed ones: then the resulting properties depend on the chosen perturbation and these studies do not describe the chaotic behaviour of the deterministic equations themselves. These approaches have known an impressive development in the recent years.

The intrinsic instability of the hydrodynamical motions, more precisely those modeled by the Euler equations (the case where the viscosity is zero) was explained geometrically without using Probability theory by V. Arnold ([3]). In his famous paper Arnold showed that the solution of Euler equation

$$
\frac{\partial u}{\partial t}+u \cdot \nabla u=-\nabla p, \operatorname{div} u=0
$$

corresponds to the velocity of a flow which is critical for the action functional

$$
S[g]=\frac{1}{2} \int\|\dot{g}(t)\|_{L^{2}}^{2} d t
$$

where $g$ denote volume preserving diffeomorphisms of the underlying manifold. Accordingly Euler equation can be regarded as the geodesic equation for the $L^{2}$ metric and one derives the instability of the motion strictly from geometrical arguments, namely from the negativity of some curvature of the space of diffeomorphisms. This is not in contradiction with the formation of the abovementionned coherent structures since Arnold describes the so-called Lagrangian flow, namely $g_{t}(x)$ such that

$$
\partial_{t} g_{t}(x)=u\left(t, g_{t}(x)\right)
$$

and not the usual Eulerian flow (of velocities). It seems that there is a kind of chaotic behaviour of the Lagrangian flow and a more predictable character of the Eulerian flow $u(t,$.$) .$

We note that this "principle" seems to apply as well to properties like ergodicity. Indeed, there are reasons to believe that the flow of velocities is ergodic (cf. [4] or a more recent discussion in 11]), invariant measures can be constructed (cf. [2] and references therein for the two-dimensional periodic case). On the other hand the Lagrangian flow is not ergodic; this has been proved in 7, for the periodic $n$-dimensional case.

The statistical effects observed in the evolution of the system are, on the other hand, related to the large number of degrees of freedom in presence, a situation quite distinct from the dynamical systems studied by Poincaré or Lorenz. 
Hydrodynamics, probability and the geometry of the diffeomorphisms group 3

Following an idea of Yasue etal ([14, 17]), consisting in interpreting the Laplacian term of Navier-Stokes equation as origin of the random behaviour of fluid particles and the drift as an avereged velocity on the stochastic (Lagrangian) motion, one is able to

a) obtain an intrinsic probabilistic representation of Navier-Stokes solution;

b) derive it as a solution of a (stochastic) variational principle;

c) deduce the corresponding (stochastic) equation for geodesics, the associated Jacobi equation and therefore consider the stability properties of the motion.

The methodology thus generalize probabilistically Arnold's approach to the case where viscosity is not zero.

\section{Geometry of the diffeomorphisms group}

Let $G_{V}$ denote the group of volume preserving homeomorphisms on a manifold. Throughout this paper we consider this manifold to be $T^{2}$, the two dimensional torus, for simplicity, since we can as well treat the torus in any dimension (cf. the remark after Theorem 2.21). On a general manifold the formulation of our results may be a more delicate matter.

The tangent space of the group $G_{V}$ will be denoted by $\mathcal{G}_{V}$; it consists of vector fields with zero divergence. The $L^{2}$ norm defines on $\mathcal{G}_{V}$ a canonical Hilbert structure. More generally, if $G^{s}=\left\{g: T^{2} \rightarrow T^{2}\right.$ bijection, $\left.g, g^{-1} \in H^{s}\right\}$ then for $s>2, G^{s}$ is a $C^{\infty}$ infinite dimensional Hilbert manifold (cf. [9, 16]). The associated tangent space, endowed with the $H^{s}$ norm, will be denoted by $\mathcal{G}^{s}$ and the tangent space for the corresponding volume preserving subgroup $G_{V}^{s}$ by $\mathcal{G}_{V}^{s}$.

We shall consider Fourier developments, namely, for $f \in L^{2}$,

$$
f(\theta)=2 \sum_{k \in \tilde{Z}^{2}} \Re \hat{f}(k) \cos (k \cdot \theta)-\Im \hat{f}(k) \sin (k \cdot \theta)
$$

where $\hat{f}$ is the Fourier transform of $f$,

$$
\hat{f}(k)=\frac{1}{(2 \pi)^{2}} \int_{T^{2}} \exp i(k . \theta) d \theta
$$

and

$$
\tilde{Z}^{2}=\left\{k=\left(k_{1}, k_{2}\right) \in Z^{2}: k_{1}>0 \text { or } k_{1}=0, k_{2}>0\right\}
$$

Notice that $f$ is real iff $\hat{f}(-k)=\overline{\hat{f}}(k)$.

We take for orthonormal basis of $\mathcal{G}_{V}^{s}$ the following vector fields:

$$
\begin{aligned}
& \left.A_{k}^{s}=\frac{1}{|k|^{s+1}}\left[\left(k_{2} \cos k \cdot \theta\right) \partial_{1}-\left(k_{1} \cos k \cdot \theta\right) \partial_{2}\right)\right] \\
& \left.B_{k}^{s}=\frac{1}{|k|^{s+1}}\left[\left(k_{2} \sin k \cdot \theta\right) \partial_{1}-\left(k_{1} \sin k \cdot \theta\right) \partial_{2}\right)\right]
\end{aligned}
$$

$k \in \tilde{Z}^{2}-\{(0,0)\}$, where $|k|^{2}=k_{1}^{2}+k_{2}^{2}$ and $\partial_{i}=\frac{\partial}{\partial \theta^{2}}$, together with the constant vector fields. 
When $s=0$ we omit the corresponding index in the notation.

The constants of structure of $\mathcal{G}_{V}^{s}$ are

$$
\begin{aligned}
{\left[A_{k}^{s}, A_{l}^{s}\right] } & =\frac{[k, l]}{2|k|^{s+1}|l|^{s+1}}\left(|k+l|^{s+1} B_{k+l}^{s}+|k-l|^{s+1} B_{k-l}^{s}\right) \\
{\left[B_{k}^{s}, B_{l}^{s}\right] } & =-\frac{[k, l]}{2|k|^{s+1}|l|^{s+1}}\left(|k+l|^{s+1} B_{k+l}^{s}-|k-l|^{s+1} B_{k-l}^{s}\right) \\
{\left[A_{k}^{s}, B_{l}^{s}\right] } & =-\frac{[k, l]}{2|k|^{s+1}|l|^{s+1}}\left(|k+l|^{s+1} A_{k+l}^{s}-|k-l|^{s+1} A_{k-l}^{s}\right) \\
{\left[\partial_{i}, A_{k}^{s}\right] } & =-k_{i} B_{k}^{s} \\
{\left[\partial_{i}, B_{k}^{s}\right] } & =k_{i} A_{k}^{s}
\end{aligned}
$$

One can find a detailed proof of these expressions in 6] for the case where $s=0$, a computation that extends easily to the general case. For the torus of higher dimensions we refer to [7.

Define the following functions

$$
\begin{aligned}
\alpha_{k, l}^{s}: & =\frac{1}{4(|k||l||k+l|)^{s+1}}\left(|k+l|^{2(s+1)}-|k|^{2(s+1)}+|l|^{2(s+1)}\right) \\
\beta_{k, l}^{s}: & =\frac{1}{4(|k||l||k-l|)^{s+1}}\left(|k-l|^{2(s+1)}-|k|^{2(s+1)}+|l|^{2(s+1)}\right) \\
{[k, l] } & =k_{1} l_{2}-k_{2} l_{1}
\end{aligned}
$$

The Christoffel symbols for the Levi-Civita connection associated to the $H^{s}$ metric can be explicitly computed; they are given by

$$
\begin{aligned}
& \Gamma_{A_{k}^{s}, A_{l}^{s}}^{s}=[k, l]\left(\alpha_{k, l}^{s} B_{k+l}^{s}+\beta_{k, l}^{s} B_{k-l}^{s}\right) \\
& \Gamma_{B_{k}^{s}, B_{l}^{s}}^{s}=[k, l]\left(-\alpha_{k, l}^{s} B_{k+l}^{s}+\beta_{k, l}^{s} B_{k-l}^{s}\right) \\
& \Gamma_{A_{k}^{s}, B_{l}^{s}}^{s}=[k, l]\left(-\alpha_{k, l}^{s} A_{k+l}^{s}+\beta_{k, l}^{s} A_{k-l}^{s}\right) \\
& \Gamma_{B_{k}^{s}, A_{l}^{s}}^{s}=[k, l]\left(-\alpha_{k, l}^{s} A_{k+l}^{s}-\beta_{k, l}^{s} A_{k-l}^{s}\right)
\end{aligned}
$$

In the case where $s=0$ the proof can be found in 6 .

A similar computation for the space $\operatorname{Diff}\left(S^{1}\right)$ was done in [1].

In order to study the geodesics corresponding to the Euler equation in fact only this case is (physically) relevant: the $L^{2}$ norm (i.e., the energy) has to be considered, even if one is lead, for technical reasons, to consider the equations in higher order Sobolev spaces, where the $L^{2}$ norm gives rise to weak Riemannian structures (cf. [9], 16]).

Concerning the corresponding Ricci curvature (for the $H^{s}$ metric) we have the following explicit expression

$$
\begin{aligned}
\operatorname{Ricci}_{s}\left(A_{j}^{s}\right)= & -2 \sum_{i}[i, j]^{2}\left(\alpha_{i, j}^{s} \alpha_{j, i}^{s}+\beta_{i, j}^{s} \beta_{j, i}^{s}\right) A_{j}^{s} \\
& -2 \sum_{i}[i, j]^{2} \frac{|i+j|^{s+1}}{(|i||j|)^{s+1}} \beta_{i+j, i}^{s} A_{j}^{s}-2 \sum_{i}[i, j]^{2} \frac{|i-j|^{s+1}}{(|i||j|)^{s+1}} \beta_{i-j, i}^{s} A_{j}^{s}
\end{aligned}
$$


Hydrodynamics, probability and the geometry of the diffeomorphisms group 5

and an analogous one for $\operatorname{Ricci}_{s}\left(B_{j}^{s}\right)$ (the curvature is a diagonal operator).

This result was proved in [7] for the case $s=0$. We notice that, in this case, the last two terms in each of the Ricci curvature expressions are symmetric,

$$
\frac{|i+j|}{|i||j|} \beta_{i+j, i}+\frac{|i-j|}{|i||j|} \beta_{i-j, i}=\frac{-(i \mid j)}{|i|^{2}|j|^{2}}+\frac{(i \mid j)}{|i|^{2}|j|^{2}}=0
$$

and we are there left with only the first terms, which reduce to:

$$
\begin{aligned}
& \operatorname{Ricci}\left(A_{j}\right)=-\sum_{i}[i, j]^{4} \frac{\left(|i|^{2}+|j|^{2}\right)}{|i|^{2}|j|^{2}|i-j|^{2}|i+j|^{2}} A_{j} \\
& \operatorname{Ricci}\left(B_{j}\right)=-\sum_{i}[i, j]^{4} \frac{\left(|i|^{2}+|j|^{2}\right)}{|i|^{2}|j|^{2}|i-j|^{2}|i+j|^{2}} B_{j} .
\end{aligned}
$$

We observe that the Ricci $_{s}$ tensors are given by divergent quantities.

On the Lie algebra $\mathcal{G}_{V}^{s}$ we may define the following process

$$
d x(t)=\sum_{k \neq 0}\left(A_{k}^{s} d x_{k}(t)+B_{k}^{s} d y_{k}(t)\right)
$$

with $s \geq 2$, where $x_{k}, y_{k}$ are independent copies of real Brownian motions. The stochastic flow

$$
\begin{aligned}
d g(t) & =(o d x(t))(g(t)) \\
g(0) & =e
\end{aligned}
$$

( $e$ being the identity) is a well defined stochastic flow of diffeomorphisms (cf. [12] and [13, 5] for exponents $s$ non necessarily greater then 2). Its generator is

$$
L^{s}=\frac{1}{2} \sum_{k \neq 0} \partial_{A_{k}^{s}} \partial_{A_{k}^{s}} F(g)+\frac{1}{2} \sum_{k \neq 0} \partial_{B_{k}^{s}} \partial_{B_{k}^{s}} F(g) .
$$

Theorem 2.1. The process $g(t)$ coincides with the Brownian motion associated to the metric $H^{s}$.

The proof follows from the explicit expression of the Christoffel symbols, more precisely from the fact that $\Gamma_{A_{k}^{s}, A_{k}^{s}}^{s}=\Gamma_{B_{k}^{s}, B_{k}^{s}}^{s}=0$.

The Laplace-Beltrami operator on vector fields $Z$ reads,

$$
\mathcal{L}^{s} Z=\frac{1}{2} \sum_{k \neq 0} \nabla_{A_{k}^{s}}^{s} \nabla_{A_{k}^{s}}^{s} Z+\frac{1}{2} \sum_{k \neq 0} \nabla_{B_{k}^{s}}^{s} \nabla_{B_{k}^{s}}^{s} Z .
$$

where $\nabla^{s}$ denotes the covariant derivative for the $H^{s}$ metric.

Then we have the following fundamental result that enables us to relate the classical Laplacian term in Navier-Stokes equations to the stochastic geometry on the space of (volume preserving) diffeomorphisms: 
Theorem 2.2. ([5]) When computed on functionals of the form $F(g)(\theta)=f(g(\theta))$, $f$ smooth, we have

with $c=\sum_{k \neq 0} \frac{\left(k_{i}\right)^{2}}{|k|^{2(s+1)}}$.

$$
L^{s} F(g)=\frac{c}{2} \Delta f
$$

The proof can be found in [5] (c.f. also [8]).

Remark 2.3. We mention that most of the results stated in this paper extend without too much trouble (but with a certain amount of work) to the case of a torus of dimension higher then two, once we choose a suitable basis of $\mathcal{G}_{V}$ and the corresponding ones for the higher order Sobolev spaces.

Denote by $\tilde{Z}^{d}$ a subset of $Z^{d}$ such that each equivalence class of the equivalence relation defined by $k \simeq k^{\prime}$ if $k+k^{\prime}=0$ has a unique representative in $\tilde{Z}^{d}$. We should take, for every $k \neq 0$, an orthonormal basis of $\left\{x \in R^{d}: k \cdot x=0\right\}$, namely $\epsilon_{k, \alpha}, \alpha=1, \ldots, d$ and define

$$
\begin{aligned}
& A_{k, \alpha}=\epsilon_{k, \alpha} \cos (k . \theta) \\
& B_{k, \alpha}=\epsilon_{k, \alpha} \sin (k . \theta)
\end{aligned}
$$

to obtain (after normalization) the corresponding basis of $\mathcal{G}_{V}^{s}$.

Furthermore we should assume that $\sum_{k, \alpha}\left[\left(\epsilon_{k, \alpha}\right)^{i}\right]^{2}$ is independent of $i$ and that $\left.\sum_{k, \alpha}\left[\left(\epsilon_{k, \alpha}\right)^{i}\right)\left(\epsilon_{k, \alpha}\right)^{j}\right)=0$ for $i \neq j$ in order to obtain the generalization of last theorem.

For the generalization of some of the results in this paper to torus of dimensions bigger then 2 we refer to [7] and to [8].

For regularity reasons, in order to avoid geometrical divergent quantities such as the Ricci tensors, we are going from now on to consider a truncated Brownian motion $x^{N}(t)$ defined as

$$
d x^{N}(t)=\sum_{0<|k| \leq N}\left(A_{k}^{s} d x_{k}(t)+B_{k}^{s} d y_{k}(t)\right)
$$

and the corresponding process $g^{N}(t)$ with generator $L^{s, N}$. Then, by the same arguments of Theorem 2.2. we can show that, when computed on functionals of the form $F(g)(\theta)=f(g(\theta)), f$ smooth, we have

$$
L^{s, N} F(g)=\frac{c_{N}}{2} \Delta f
$$

with $c_{N}=\sum_{0<|k| \leq N} \frac{\left(k_{i}\right)^{2}}{|k|^{2(s+1)}}$.

\section{A variational principle}

For a time dependent vector field $u \in L^{2}\left([0, T] ; \mathcal{G}_{V}\right)$, let $g_{u}^{N}$ be a solution of the s.d.e. with values in $G_{V}$, 
Hydrodynamics, probability and the geometry of the diffeomorphisms group 7

$$
d g_{u}^{N}(t)=\left(u(t) d t+\sqrt{\frac{\nu}{c_{N}}} o d x^{N}(t)\right) g_{u}^{N}(t), g_{u}(0)=e
$$

with generator

$$
L_{u}^{s, N} F(g)=\frac{\nu}{c_{N}} L^{s, N} F+\sum_{k} u^{A_{k}^{s}} \partial_{A_{k}^{s}} F+\sum_{k} u^{B_{k}^{s}} \partial_{B_{k}^{s}} F .
$$

A weak solution of such stochastic differential equation can be shown to exist (cf. [5).

Then, when computed on functionals of the form $F(g)(\theta)=f(g(\theta)), f$ smooth, we have

$$
L_{u}^{s} F(g)=\nu \Delta f+u . \nabla f .
$$

Notice that the constant appearing in the diffusion coefficient is chosen to produce, after "projection" on pointwise functions, the viscosity parameter $\nu$ in front of the Laplacian.

For smooth functionals $F(g, t)$ denote

$$
D F(g(t), t)=\lim _{\epsilon \rightarrow 0} E^{\mathcal{P}_{t}}(F(g(t+\epsilon), t+\epsilon)-F(g(t), t))
$$

For (time dependent) vector fields $Z(t,.) \in \mathcal{G}_{V}$, and using the Itô parallel transport (associated to the Levi-Civita connection) $\mathcal{T}_{s \leftarrow t}$ above the process $g(t)$, which is the solution of the stochastic differential equation

$$
\begin{aligned}
d_{t}\left[\mathcal{T}_{s \leftarrow t}\right] Z_{t} & =-\left(\Gamma_{k}\right)_{g(t)} o d x_{t}^{k}\left[\mathcal{T}_{s \leftarrow t}\right] Z_{t} \\
{\left[\mathcal{T}_{s \leftarrow s}\right] } & =I d
\end{aligned}
$$

define

$$
\left.D Z(g(t), t)=\lim _{\epsilon \rightarrow 0} \frac{1}{\epsilon} E^{\mathcal{P}_{t}}\left(\mathcal{T}_{t \leftarrow(t+\epsilon)} Z(g(t+\epsilon), t+\epsilon)-Z(g(t), t)\right)\right) .
$$

Consider now the following (stochastic) action functional

$$
S[g]=\frac{1}{2} E \int_{0}^{T}\|D g(t)\|_{L^{2}}^{2} d t-\frac{1}{2} E\|D g(0)\|_{L^{2}}^{2}
$$

The Navier-Stokes solution can be characterized as the drift of a critical process for this action. More precisely we have,

Theorem 3.1. ( 5 )

The process $g_{u}^{N}(t)$ is a critical point of the energy functional $S$ if and only if $u$ verifies the $N$. S. equation

$$
\begin{array}{r}
\frac{\partial u}{\partial t}+u . \nabla u=\nu \Delta u-\nabla p \\
\operatorname{div} u=0, u(T, \theta)=u_{T}(\theta)
\end{array}
$$


We precise that critical here means in the space of continuous $G_{V}$-valued semi-martingales $g(t)$ with $g(0)=e$ with variations defined as

$$
\left(D_{l}\right) S[e .(v)]=\left.\frac{d}{d \epsilon} S[e .(v) o g(.)]\right|_{\epsilon=0}
$$

where

$$
\begin{array}{r}
e_{t}(v), v \in C^{1}\left([0, T] ; \mathcal{G}_{V}^{\infty}\right), v(0, .)=0 \\
\frac{d e_{t}(v)}{d t}=\dot{v}\left(t, e_{t}(v)\right), e_{0}(v)=e .
\end{array}
$$

\section{Stochastic geodesics equation}

To a variational principle a Euler-Lagrange dynamics can be associated. In this framework the corresponding equations are stochastic and correspond to a "regularized" version of the equation of geodesics for the $L^{2}$ metric.

Writting $e_{k}$ for a generic element of the basis of $\mathcal{G}_{V}^{s}\left(A_{k}^{s}\right.$ or $\left.B_{k}^{s}\right)$ and $u^{k}=u^{e_{k}}$, define the following truncated approximation of the Ricci $_{s}$ tensor

$$
\begin{aligned}
R_{s}^{N}(\tilde{u})= & \sum_{0<|k| \leq N} \sum_{l, m} \Gamma_{e_{k}, e_{l}}^{s}\left(\Gamma_{e_{m}, e_{k}}^{s} \mid e_{l}\right) \tilde{u}^{m}-\left(\left[e_{k}, e_{m}\right] \mid e_{l}\right) \Gamma_{l, k}^{s} \tilde{u}^{m} \\
= & -2 \sum_{0<|k| \leq N} \sum_{m}[k, m]^{2}\left(\alpha_{k, m}^{s} \alpha_{m, k}^{s}+\beta_{k, m}^{s} \beta_{m, k}^{s}\right) \tilde{u}^{m} \\
& -4 \sum_{0<|k| \leq N}[k, m]^{2} \frac{|k+m|^{s+1}}{(|k||m|)^{s+1}} \beta_{k+m, k}^{s} \tilde{u}^{m}
\end{aligned}
$$

Theorem 4.1. Let $s \geq 0$. The vector field $\tilde{u}(t, \theta)=-u((T-t), \theta)$ is a solution of the Navier-Stokes equation if and only if $u$ is a martingale of the process $g_{u}^{N}$.

Equivalently,

$$
D D g_{u}^{N}(t)-\frac{\nu}{2 c} R_{s}^{N}\left(D g_{u}^{N}\right)=0 \text { a.e. }
$$

with

$$
g_{u}^{N}(0)=e, D g_{u}^{N}(T)=u\left(T, g_{u}^{N}(T)\right)
$$

or

$$
\frac{\partial \tilde{u}}{\partial t}+\tilde{u}^{k} \nabla_{e_{k}} \tilde{u}-\mathcal{L}^{s, N} \tilde{u}+\frac{\nu}{2 c_{N}} R_{s}^{N}(\tilde{u})=0
$$

Remark 4.2.

1. The presence of the truncated Ricci term is due to the choice of the Itô stochastic parallel transport (associated with the Levi-Civita connection). This term disappears if we define the operator $D$ using the so-called "damped" parallel transport, which incorporates the effect of the curvature. 
Hydrodynamics, probability and the geometry of the diffeomorphisms group 9

2. The covariant derivation appearing in the last formula of the Theorem is the one associated to the $L^{2}=H^{0}$ metric, as it should be, since it is the (Euler) non linear term of the equation. On the other hand the stochastic perturbations of the Euler equation, responsible for the second order term of the Navier-Stokes equation, are related to the $H^{s}$ metric.

3. The expression

$$
\mathcal{L}^{s, N} \tilde{u}-\frac{\nu}{2 c_{N}} R_{s}^{N}(\tilde{u})=\nu \Delta \tilde{u}
$$

may be well defined with each of its separate terms divergent, depending on the regularity of the Navier-Stokes velocity function $u$.

Proof. The term $\tilde{u}^{k} \nabla_{e_{k}} \tilde{u}$ corresponds, as said before, to the non-linear part of the Navier-Stokes equation; so, in this proof, we will be analysing the term coming from the stochastic perturbation and, for simplicity, we omit the index $s$ in the corresponding Christoffel symbols.

Writing

$$
\begin{aligned}
\nabla_{e_{k}}^{s} \nabla_{e_{k}}^{s} \tilde{u} & =\partial_{e_{k}}\left(\nabla_{e_{k}}^{s} \tilde{u}\right)+\Gamma_{k, l}\left(\nabla_{e_{k}}^{s} \tilde{u}\right)^{l} \\
& =\partial_{e_{k}} \partial_{e_{k}} \tilde{u}+\Gamma_{k, l} \Gamma_{k, m}^{l} \tilde{u}^{m}
\end{aligned}
$$

we have

$$
\begin{aligned}
& \sum_{0<|k| \leq N} \nabla_{e_{k}}^{s} \nabla_{e_{k}}^{s} \tilde{u}-\partial_{e_{k}} \partial_{e_{k}} \tilde{u}-R_{s}^{N}(\tilde{u}) \\
= & \sum_{0<|k| \leq N} \sum_{l, m} \Gamma_{k, l}\left[e_{k}, e_{m}\right]^{l} \tilde{u}^{m}-\Gamma_{l, k}\left[e_{k}, e_{m}\right]^{l} \tilde{u}^{m} \\
= & \sum_{0<|k| \leq N} \sum_{l, m}\left[e_{k}, e_{l}\right]\left[e_{k}, e_{m}\right]^{l} \tilde{u}^{m}
\end{aligned}
$$

We analyse the component in $B_{m}^{s}$ (the one in $A_{m}^{s}$ will give a similar result).

We have to sum the last expression in the indices $k$ and $l$. The two non zero contributions come from

$$
\begin{aligned}
I & =\left[A_{k}^{s}, B_{m}^{s}\right]^{A_{l}^{s}}\left[A_{k}^{s}, A_{l}^{s}\right] \tilde{u}^{B_{m}^{s}} \\
I I & =\left[B_{k}^{s}, B_{m}^{s}\right]^{B_{l}^{s}}\left[B_{k}^{s}, B_{l}^{s}\right] \tilde{u}^{B_{m}^{s}}
\end{aligned}
$$

Computation of $I$ :

When $l=k+m$,

$$
\begin{aligned}
I= & -\frac{[k, m]}{2|k|^{s+1}|m|^{s+1}}|k+m|^{s+1} \frac{[k, k+m]}{2|k|^{s+1}|k+m|^{s+1}}\left(|2 k+m|^{s+1} B_{2 k+m}^{s}\right. \\
& \left.+|m|^{s+1} B_{m}^{s}\right) \tilde{u}^{B_{m}^{s}} \\
= & -\frac{[k, m]^{2}}{4|k|^{2(s+1)}|m|^{s}}\left(|2 k+m|^{s+1} B_{2 k+m}^{s}+|m|^{s+1} B_{m}^{s}\right) \tilde{u}^{B_{m}^{s}}
\end{aligned}
$$


and, when $l=k-m$,

$$
\begin{aligned}
I= & -\frac{[k, m]}{2|k|^{s+1}|m|^{s+1}}|k-m|^{s+1} \frac{[k, m]}{2|k|^{s+1}|k-m|^{s+1}}\left(|2 k-m|^{s+1} B_{2 k-m}^{s}\right. \\
& \left.+|m|^{s+1} B_{m}^{s}\right) \tilde{u}^{B_{m}^{s}} \\
= & -\frac{[k, m]^{2}}{4|k|^{2(s+1)}|m|^{s+1}}\left(|2 k-m|^{s+1} B_{2 k-m}^{s}+|m|^{s+1} B_{m}^{s}\right) \tilde{u}^{B_{m}^{s}}
\end{aligned}
$$

(notice that $B_{-m}^{s}=B_{m}^{s}$ ).

Concerning $I I$, we have, for $l=k+m$,

$$
I I=\frac{[k, m]^{2}}{4|k|^{2(s+1)}|m|^{s+1}}\left(|2 k+m|^{s+1} B_{2 k+m}^{s}-|m|^{s+1} B_{m}^{s}\right) \tilde{u}^{B_{m}^{s}}
$$

and, for $l=k-m$,

$$
I I=\frac{[k, m]^{2}}{4|k|^{2(s+1)}|m|^{s+1}}\left(|2 k-m|^{s+1} B_{2 k-m}^{s}-|m|^{s} B_{m}^{s}\right) \tilde{u}_{m}^{B_{m}^{s}}
$$

Summing up in $k$ the non diagonal terms cancel and

$$
\begin{aligned}
& \sum_{0<|k| \leq N}\left(\nabla_{e_{k}}^{s} \nabla_{e_{k}}^{s} \tilde{u}\right)^{B_{m}^{s}}-\left(R_{s}^{N} \tilde{u}\right)^{B_{m}^{s}}=-\sum_{k} \frac{[k, m]^{2}}{2|k|^{2(s+1)}} \tilde{u}^{B_{m}^{s}} \\
= & -\sum_{k} \frac{k_{1}^{2} m_{2}^{2}}{2|k|^{2(s+1)}} \tilde{u}^{B_{m}^{s}}-\sum_{k} \frac{k_{2}^{2} m_{1}^{2}}{2|k|^{2(s+1)}} \tilde{u}_{m}^{B_{m}^{s}}+\sum_{k} \frac{k_{1} m_{2} k_{2} m_{1}}{2|k|^{2(s+1)}} \tilde{u}_{m}^{B_{m}^{s}}
\end{aligned}
$$

Since $c_{N}=\sum_{0<|k| \leq N} \frac{k_{1}^{2}}{|k|^{2(s+1)}}=\sum_{0<|k| \leq N} \frac{k_{2}^{2}}{|k|^{2(s+1)}}$,

$$
\sum_{0<|k| \leq N} \frac{1}{2}\left(\left(\nabla_{e_{k}}^{s} \nabla_{e_{k}}^{s} \tilde{u}\right)^{B_{m}^{s}}-\frac{\nu}{c_{N}}\left(R_{s}^{N} \tilde{u}\right)^{B_{m}^{s}}\right)=-\nu m^{2} \tilde{u}_{m}^{B_{m}^{s}}
$$

that is, we have the spectral decomposition of the Laplace operator, and the result follows.

The first statement of the theorem, namely the characterization of $u\left(g_{u}^{N}\right)$ as a martingale, comes from Itô formula and the fact that the bounded variation part of this process is precisely the expression $\frac{\partial \tilde{u}}{\partial t}+\tilde{u}^{k} \nabla_{e_{k}} \tilde{u}-\mathcal{L}^{s, N} \tilde{u}+\frac{\nu}{2 c_{N}} R_{s}^{N}(\tilde{u})$.

\section{Final comments}

The probabilistic characterization of the Navier-Stokes solutions described above allows to study various of their properties. But one can legitemely ask wether it shares any light on the construction of the solutions itself. This would amount to ask wether we can solve directly (and in a probabilistic way) the stochastic equation

$$
D D g_{u}^{N}(t)+\frac{\nu}{2 c_{N}} R_{s}^{N}\left(D g_{u}^{N}\right)=0
$$


Hydrodynamics, probability and the geometry of the diffeomorphisms group 11

Stated like this, we do not have a straightforward answer. Nevertheless one can use another probabilistic characterization, in terms of forward-backward stochastic equations, based on the observation that the process $g_{u}^{N}(t)$ satisfies not only

$$
d g_{u}^{N}(t)=\left(u_{t} d t+\sqrt{\frac{\nu}{c_{N}}} o d x_{N}(t)\right) g_{u}^{N}(t), g_{u}^{N}(0)=e
$$

but also the equation

$$
d u_{t}=-\nabla p\left(t, g_{u}^{N}(t)\right) d t+X_{t} d x_{N}(t), D g_{u}^{N}(T)=u\left(T, g_{u}^{N}(T)\right)
$$

Considering these two equations as a system on $\left(g_{u}^{N}(t), u_{t}, X_{t}\right)$ we have a forward-backward system to solve (analytically and numerically) (cf. [8] and work in progress).

\section{References}

[1] H. Airault, P. Malliavin, Quasi-invariance of Brownian measures on the group of circle homeomorphisms and infinite-dimensional Riemannian geometry.J. Funct. Anal. 241 (2006), 99-142.

[2] A. Albeverio, A.B. Cruzeiro, Global flows with invariant (Gibbs) measures for Euler and Navier-Stokes two dimensional fluids. Comm. Math. Phys. 129 (1990), 431-444.

[3] V.I. Arnold, Sur la géométrie différentielle des groupes de Lie de dimension infinie et ses aplications à l' hidrodynamique des fluides parfaits. Ann. Inst. Fourier 16 (1966), 316-361.

[4] G.K. Batchelor, The theory of homogeneous turbulence. Cambridge Monographs on Mechanics \& Applied Math. Series, 1953.

[5] F. Cipriano, A.B. Cruzeiro, Navier-Stokes equation and diffusions on the group of homeomorphisms of the torus. Comm. Math. Phys. 275 (2007), 255-269.

[6] A.B. Cruzeiro, F. Flandoli, P. Malliavin, Brownian motion of volume preserving diffeomorphisms and existence of global solutions of $2 D$ stochastic Euler equation. J. Funct. Anal. 242 (2007), 304-326.

[7] A.B. Cruzeiro, P. Malliavin, Nonergodicity of Euler fluid dynamics on tori versus positivity of the Arnold-Ricci tensor. J. Funct. Anal. 254 (2008), 1903-1925.

[8] A.B. Cruzeiro, E. Shamarova, Navier-Stokes equations and forward-backward SDEs on the group of diffeomorphisms of a torus. http://arXiv.org/abs/0807.0421

[9] D. Ebin, J. Marsden, Groups of diffeomorphisms and the motion of an incompressible fluid. Ann. of Math. 92(1) (1970), 102-163.

[10] A.V. Fursikov, A.I. Komech, M.I. Vishik, Some mathematical problems of statistical hydrodynamics. Dokl. Akad. Nauk. SSSR 246(5) (1979), 1037-1041.

[11] R. Galanti, A. Tsinober, Is turbulence ergodic?. Phys. Letters A 330 (2004), 173-180.

[12] H. Kunita, Stochastic flows and stochastic differential equation. Cambridge University Press, 1990.

[13] P. Malliavin, The canonic diffusion above the diffeomorphism group of the circle. C.R. Acad. Sci. Paris 329 Ser. I (1999), 325-329. 
[14] T. Nakagomi, K. Yasue, J.C. Zambrini, Stochastic variational derivations of the Navier-Stokes equation. Lett. Math. Phys. 160 (1999), 337-365.

[15] R. Robert, L'effet papillon n'existe plus!. SMF-Gazette-90, 2001.

[16] S. Skholler, Geometry and curvature of diffeomorphism group with $H^{1}$ metric and mean hydrodynamics. J. Funct. Anal. 160(1) (1998), 337-365.

[17] K. Yasue, A variational principle for the Navier-Stokes equation. J. Funct. Anal. 51(2) (1983), 133-141.

\author{
Ana Bela Cruzeiro \\ Dep. Matemática I.S.T. (TUL) \\ Av. Rovisco Pais \\ 1049-001 Lisboa \\ Portugal \\ \& \\ Grupo de Física-Matemática da Universidade de Lisboa \\ Av. Prof. Gama Pinto, 2 \\ 1649-003 Lisboa \\ Portugal \\ e-mail: abcruz@math.ist.utl.pt
}

